\title{
Validation of automated apparatus for upper limb velocity testing
}

\author{
Leandro Medeiros da Silva ${ }^{1 *}$, Felipe José Aidar², Dihogo Gama de Matos², Eduardo Estevan \\ Santana ${ }^{2}$, Matheus Peixoto Dantas ${ }^{1}$, Petrus Gantois Massa Dias dos Santos ${ }^{3}$, Paulo Moreira \\ Dantas $^{2}$, Breno Guilherme de Araújo Tinôco Cabral ${ }^{2}$
}

\begin{abstract}
The purpose of this paper was to validate the ability of automated devices to measure the velocity of the upper limbs. Thus, 144 students, from 8 to 17 years old, both sexes, participated in the study in a social project in the city of Natal/RN. The Automated Tapping Test (AATT) was developed following the dimensions of the original EUROFIT test, but built out of metal parts, so that the calibration was more accurate and could be read as an electronic signal by the integrated circuit. The upper limb velocity test was applied in three steps: before performing the original test, all participants underwent a laterality check and then motor practice similar to the original test. After 30 minutes, the first step in the pattern established by the EUROFIT test battery was performed and 30 minutes later, the third stage, with the AATT. The measurement reliability of the automated apparatus was tested through intraclass correlations and a strong intraclass interaction coefficient of $r=0.901$ was found. In conclusion, the AATT was found to be accurate and efficient as to what it is proposed to evaluate, being able to minimize the intra-evaluator error and to assist professionals in the sports.

Keywords: device validation, motor evaluation, plate strike, tapping test, velocity of upper limbs.
\end{abstract}

\section{INTRODUCTION}

There has been increasing use of test batteries to evaluate physical conditions in the elderly (Rickli \& Jones, 1999), adults (Marques et al., 2014) and children (Alves et al., 2014). However, some criteria have to be observed, and among them establishing the reliability and objectivity of the tests stands out. Regarding the objectivity of a measuring instrument, this can be defined as the degree of precision of the results even when it is applied by different evaluators to the same individuals. In addition, the reliability or reproducibility of a measuring instrument is the degree to which the results are expected to be consistent or reproducible when performed in similar situations by the same observer at different times (Falzon et al., 2014; Wang et al., 2014).

The EUROFIT battery test (Eurofit, 1990) was developed to evaluate the velocity of the upper limbs and, according to Schmidt and Wrisberg
(2001), is classified as a continuous and closed motor task. The measurement is usually done by an evaluator and the time is recorded by a second person with a timer (Eurofit, 1990).

Researchers have focused on motor evaluation for different sports under different conditions (Brasile, 1990; Brasile, 1986, Kilkens et al., 2003), besides other physical qualities and field activities (Kilkens et al., 2003; Yilla \& Sherril, 1998), where the wide range of tasks and measurement parameters has made it difficult to analyze the evaluation process. Thus, the purpose of using an electronic touch measurement system is to visualize the intrinsic information of the test, which can only be observed with the final results.

The aim of this study was to validate an automated upper limb velocity evaluation apparatus in order to apply the test validated by EUROFIT (Eurofit, 1990) more efficiently, facilitating motor assessments by professionals and minimizing any human error.

\footnotetext{
Manuscript received at April 14 $4^{\text {th }} 2018$; Accepted at November $8^{\text {th }} 2018$

${ }^{1}$ Federal University of Rio Grande do Norte, Natal, Rio Grande do Norte, Brazil

${ }^{2}$ Federal University of Sergipe, São Cristovão, Sergipe, Brazil

${ }^{3}$ Federal University of Paraiba, João Pessoa, Brazil

* Corresponding author: Email: lndrmedeiros@gmail.com
} 


\section{METHOD}

\section{Participants}

A cross-sectional study was carried out in which children and adolescents participating in a sports initiation project were enrolled in the study. The population was composed of 144 children and adolescents of both sexes ( $n=144$; 76 boys and 68 girls), aged 8 to 17 years. The criteria for inclusion were: age compatible with the age established for the research (8-18 years), presented the Terms of Consent and Free Clarification (TCLE) signed by the responsible adult and the term of assent (TIC) signed by the child or adolescent. Exclusion criteria were: refusal to participate in the physical tests, failure to present the signed TIC or TALE, or presenting any motor or cognitive impairment that would make performing the tests impossible.

All participants were given an explanation about the performance of the tests and use of the device. A test familiarization session was carried out, consisting of (a) Original plate striking test and (b) the Automated Tapping Test (AATT), with a 30 min interval between them. Then, data collection was performed in two stages, with initially the participants performing the test according to the original protocol (Eurofit, 1990) and $30 \mathrm{~min}$ after the first test they performed the AATT. All participants received verbal stimuli during the tests.

The study was approved by the Ethics and Research Committee of the Health Sciences Center of the Federal University of Rio Grande do Norte (Opinion No. 1249937/2015), following the guidelines for data collection in human beings, according to resolution no. 466/12, of $12 / 12 / 12$, of the National Health Council, as well as the ethical principles in the Declaration of Helsinki (2008) that were strictly observed and adopted according to the drawing shown in Figure 1.

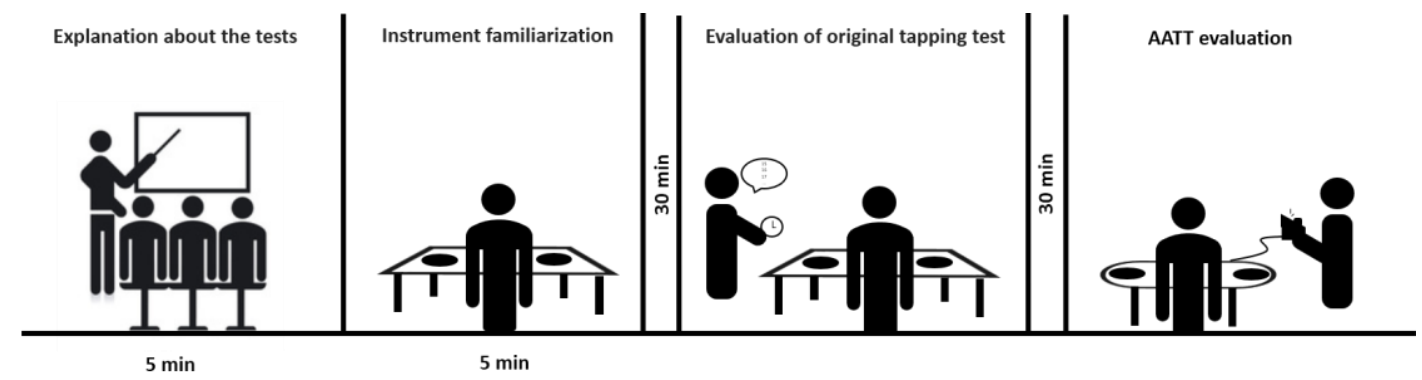

Figure 1. Study design with sequence of actions

\section{Measures}

The data collection was performed on the conditions of manual dominance and motor performance of the participants. Manual dominance was determined by means of the hand preference diagnostic test, in which the participant throws a tennis ball, writes or draws (using pen and paper), and makes a square with matchsticks, and each participant was characterizing as right handed, left-handed or undefined (Neto et al., 2013). Stroke of EUROFIT plates: The following equipment is required for the test: a) An adjustable table: the table height must be adjusted to the height of the participant's navel; b) Two disks (diameter of 20 centimeters), positioned 60 centimeters apart in a line parallel to the subject; c) A rectangle $(30 \times$
20 centimeters), positioned equidistant between both disks; the setup is shown in Figure 2.

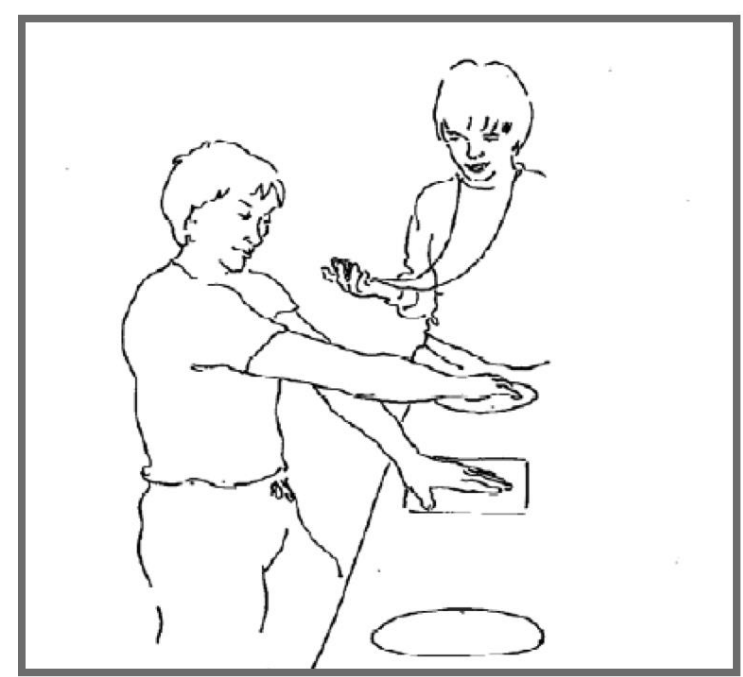

Figure 2. Tapping Test (EUROFIT,1990) 
Automated Tapping Test (AATT): this apparatus (see Figure 3) was built with the purpose of measuring data that allows for the optimization of the EUROFIT test (Eurofit, 1990). The AATT has the same dimensions as the original test, is composed of metal parts with adjustable height and distance between plates for fixed-time and age-time protocols. An electronic system of measurement and software developed for the same purposes. In this way, the system registers the performance of the touch-to-touch performer, considering the accelerations and decelerations, from the beginning of the test until the end of the test, making possible other inferences, such as contact time with the plates and the time of flight between the plates for each cycle, and the total test time with millisecond precision. The apparatus presents sound and luminous signals that aid the evaluator and signals the beginning and end of the test and automatically counts the time of accomplishment according to methodology validated in the original test.

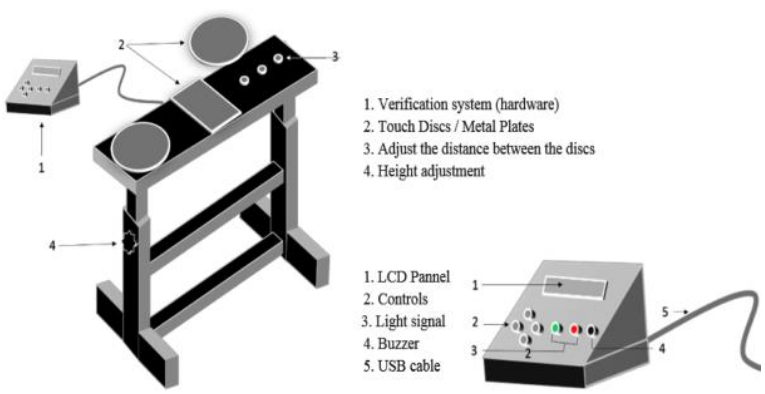

Figure 3. Automated Apparatus Tapping Test Model (AATT).

The same evaluator carried out the evaluations and the importance of this is the smaller difference makes it possible to minimize any errors in the measurement, namely the intraevaluator technical error of measurement (ETM). In order to measure the results between the different types of application of the plate striking protocol (AATT and original EUROFIT), the following equation was used to perform the calculations (Perini et al., 2005).

$$
\text { Absolut ETM }=\sqrt{\frac{\sum d_{i}^{2}}{2 n}}
$$

Where: $\Sigma=$ Sum of deviation squared; $\mathrm{n}=$ number of volunteers measured; $i=$ how many deviation

$$
\text { Relative } E T M=\frac{E T M}{V M V} \times 100
$$

Where: ETM = Technical error measurement, expressed in \%; VMV = Average value of the variable.

\section{Procedures}

Test stroke of EUROFIT plates

The participants' motor performance was verified through a velocity test of the upper limbs by means of the Plate Strike test, following the protocol of EUROFIT (Eurofit, 1990) and then evaluated using the AATT.

For the performance of the test, the evaluator controls the cycles, the total time, the beginning and the end of the test. The non-dominant hand of the appraised is supported in the central rectangle, and the appraised hand moves from one side to the other, as fast as possible to place the dominant hand on either side of the nondominant hand. This action is repeated for 25 cycles, or 50 touches on the plates (Eurofit, 1990).

For the original test, the Council of Europe recommends that in order to carry out the EUROFIT Plate Strike test, to take into account some important additional points such as the counting of the cycles, which should be out loud and done by the evaluator; the table should be at the navel height of the child; the evaluator should stand on the side of the hand for which the child will start the test; the hand in the rectangle must remain in it and the other must always touch the disks; two evaluators are recommended during the test, one to time and another to count the cycles; in case the child does not touch the disk, an extra touch must be added in order for it to reach the full 25 cycles (Eurofit, 1990).

Performance of the Automated Tapping Test (AATT)

To perform the Plate Strike test using the Automated Tapping Test (AATT), the methodological procedure was identical to the original test (EUROFIT); however, the count of 
the cycles and touches is performed by the software developed for the AATT. To perform the counting of the taps, the automated system of the AATT consists of an Arduino Uno microcontroller platform that sends an electric signal of $5 \mathrm{~V}$ to the central metal plate of the mechanical system, and a voltage comparison circuit using an integrated circuit connected to the two metal plates at the ends of the mechanical system. The end plates meet at a voltage of $0 \mathrm{~V}$. The test is initiated when the user positions the non-dominant hand on the center plate of the platform and touches the other hand on the end plate of the dominant hand side. The user has an important role because the user's body makes a connection bridge between the two boards by sending a $5 \mathrm{~V}$ voltage to the end plate to perform the voltage comparison. The voltage comparison circuit sends a voltage signal to the Arduino microcontroller platform when the end plate receives a voltage of $4 \mathrm{~V} \sim 5 \mathrm{~V}$, so the microcontroller counts the taps, the time interval and the total time of the test.

Even if the user touches the same disk twice the count only continues if there is an alternation between the touches on the disks that will send the data to the software installed on the computer connected to it. The beginning and end of the test will also be signaled by the device through a sound and luminous signal guaranteeing the attention of the evaluated by using different stimuli. In this way, only one evaluator is required to adjust the start of the procedure on the computer connected to the device.

\section{Statistical analysis}

The Kolmogorov-Smirnov test was applied to verify the assumption of normal data distribution, which was confirmed for all variables. The descriptive analysis consisted of measures of mean and standard deviation of all variables of the study. To verify the validity of the apparatus the results were evaluated compared to the mean results by Pearson's correlation coefficient test (r), the coefficient of determination $\left(\mathrm{r}^{2}\right)$. The correlation was proposed by (Lin, 1989) for an analysis of the Concordance Correlation Coefficient (CCC), in order to verify the accuracy $(\mathrm{Cb})$ and the precision $(\rho)$ between the estimated and average values in the validation. Intracanal correlation coefficient (ICC), table. The Bland-Altman plot was published to verify the result and a concordance between the original test and the AATT, with the limits of agreement (LC) defined as a mean of the differences +1.96 standard deviations. Abstract Software for the Social Sciences (SPSS), version 20.0 and MedCalc v.12.5.0.

\section{RESULTS}

Table 1 shows the descriptive results of the evaluations.

Table 1

Descriptive analysis of the evaluated ones

\begin{tabular}{|c|c|c|c|c|c|}
\hline \multicolumn{2}{|c|}{ Variables } & $\mathbf{N}$ & $(\%)$ & Mean & SD \\
\hline \multirow[t]{2}{*}{$\begin{array}{l}\text { Dominant } \\
\text { hand }\end{array}$} & $\begin{array}{l}\text { Right - } \\
\text { handed }\end{array}$ & 138 & 95,8 & - & - \\
\hline & $\begin{array}{l}\text { Left- } \\
\text { handed }\end{array}$ & 6 & 4,2 & - & - \\
\hline Age & & 144 & - & 12,61 & 0,8994 \\
\hline
\end{tabular}

The mean comparison test did not identify a significant difference between the two assessment methods $(p=0.647)$.

Table 2

Technical error of measurement (ETM) between the evaluation performed by the original protocol and use of the AATT device

\begin{tabular}{lcc}
\hline & $\begin{array}{c}\text { OriginalTest } \\
\text { (EUROFIT) }\end{array}$ & $\begin{array}{c}\text { AATT } \\
\text { Test }\end{array}$ \\
\hline $1^{\text {st }}$ evaluation & 14,04 & 13,02 \\
$2^{\text {nd }}$ evaluation & 13,20 & 12,89 \\
Mean & 13,62 & 12,94 \\
ETM & $4,8 \%$ & $1,52 \%$ \\
\hline
\end{tabular}

The technical error of intra-rater measurement was verified between the two evaluations carried out through each test, being the verified difference between them, with significantly better results for AATT, although the original test presented an acceptable classification. In order to demonstrate the ability to calibrate the instrument, with variables intrinsic to the test, three graphs were developed that demonstrate the total test time for the 50 taps, the flight time and the contact time with the plates. The mean total test time was 12.89 seconds for the original test and 12.77 seconds for the AATT. It was identified that about $67.84 \%$ 
of the total time is composed of flight time, and $32.15 \%$ of contact with the metal plates.

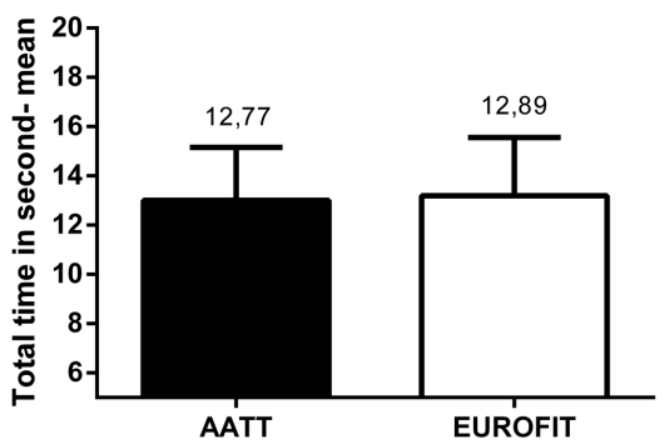

Figure 4. Average total time

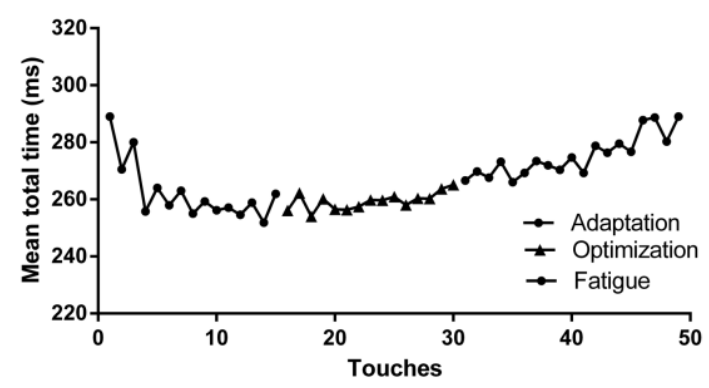

Figure 5. Average total flight time

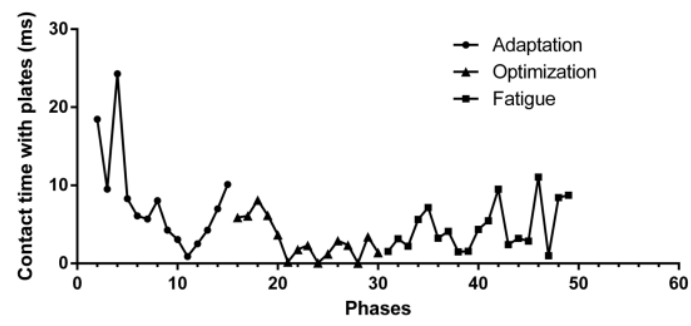

Figure 6. Average time contacts with plates

The data found in the validation analyzes show quite significant indices. A coefficient of determination $\left(\mathrm{r}^{2}\right)$ 0.716, in the analysis of the Coefficient of Correlation of Concordance (CCC) 0,823 , validity $(\mathrm{cb}) 0,999$ and precision $(\rho) 0,820$; The Bland and Altman model was used to demonstrate the intraclass correlation ICC (0.901) of the applied tests.

The Bland and Altman model was used to demonstrate the intraclass correlation of the applied tests, for which an $r=-0.901$ was found to be very high, which is presented as an $r^{2}$ of 0.711 .

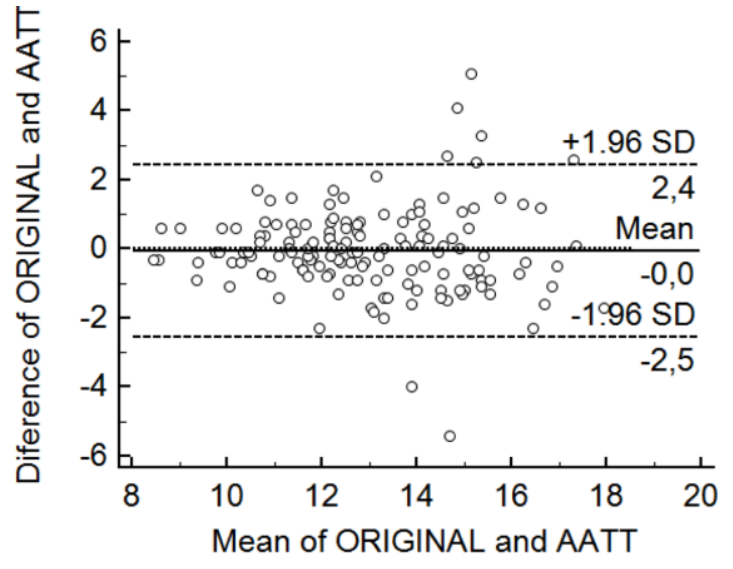

Figure 7. Intraclass correlation, Bland - Altman plot

\section{DISCUSSION}

The aim of the present study was to validate an automated upper limb velocity evaluation apparatus in order to make the application of the test validated by EUROFIT (Eurofit, 1990) more efficient, facilitating motor assessments for professionals and minimizing human error.

Thus, we validated the AATT, which proposes to assess the velocity of upper limbs, through the original Strike Plate protocol published by EUROFIT (Eurofit, 1990) after considering that the original methodology applied could be improved and optimized, facilitating the collection of data from large groups in the sports and health area. The developed device demonstrates efficiency in its results when compared with the original test.

In the development of unilateral limb protocols it is important to observe the motor preference of the individuals evaluated. There is a general preference in the population for the use of the right hemisphere to perform these tests (Neto et al., 2013).

When observing the total time of the tests, with the means presented in Figure 4, (EUROFIT $12.892 \mathrm{~s}$ and AATT $12.772 \mathrm{~s}$ ) do not present significant differences and are quite close to those of studies that used the same testing protocol. Santos (2009) presented similar averages in his studies when he performed pre and post intervention tests in youngsters between 12 and 15 years old with averages of $12.99 \mathrm{~s}$ for the group with three training days, $13.73 \mathrm{~s}$ for the group with one training day, and $13.24 \mathrm{~s}$ for the control group. Dantas et al., (2016), using the original 
protocol for groups between 12 and 15 years old, in a study that verified the relationship of bone and chronological age with physical abilities in sports initiation, found means ranging from 15.72 to $15.16 \mathrm{~s}$. These variations can be explained based on factors such as motor coordination that can suffer a deficit in the pubertal period due to the peak of physical growth that is not accompanied at the same speed by neural development, being then balanced with the somatic aspects (Dantas et al., 2016). In another study by Cabral (2013) presented similar results (mean $13.2 \mathrm{~s}$ ) in a population in the same age group.

In the original EUROFIT test, the responsibility for measuring time and cycles by one or two evaluators requires unnecessary wear, since they must count aloud and be synchronized to the time/touch during the test. This may compromise the data in collections from large groups and may increase the intra-evaluative error or inter-rater errors. We verified the difference in the results between the two types of procedure evaluated in this study, with the MES in the original procedure 4.8 vs. $1.52 \mathrm{~s}$ in the protocol evaluated by the AATT apparatus. Therefore, the creation of an automated device reduces the chances of human error. In addition, it allows for standardization of the processes, greater efficiency in testing, and, often, reductions in costs and time (Cabral, 2013).

In this context, Santos (2009) presented a device intended to measure upper limb velocity measurements. According to these authors, the creation of a device used to measure the velocity of upper limbs proved to be very efficient because with the instrument developed for their research it was possible to record performance curves of the test for each subject, and these curves could be analyzed in relation to the total test time, flight time between the touches on the plates, and the contact time of the hand with each plate.

The use of other devices for measuring upper limb velocity has been shown to be efficient when used in collection of data from children of different ages (Santos et al., 2007; Krebs, Borges Junior \& Santos, 2008; Krebs, Lucas \& Saens, 2000), demonstrating the effectiveness of the measures recorded. Krebs et al., (2008) used an apparatus for measuring the upper limb velocity of six university basketball players, with a system of plates and an analog converter following the protocol of EUROFIT (Eurofit, 1990). Although it was not completely objective, the equipment used proved to have been quite efficient in the data collection, and they had the intention to also verify proprioceptive visual ability.

It is important to emphasize that the application of the AATT follows the same protocol and requirements proposed by the European Commission (Eurofit, 1990) to evaluate the velocity of upper limbs, following the height adjustment of the support table, the proposed dimensions and an execution identical to the original protocol. In addition, the AATT allows for adjustment of the distance between the disks as well as the table height, which corroborates Finamor's studies. Krebs et al., (2008) analyzed aspects of physical development (size and height) related to structural dimensions, execution and performance in the Plate Strike test. In addition, they evaluated the correlation of plate distances in indoor soccer and basketball practitioners and identified a moderate negative relationship between the original plate striking test and the fit test between plate distances in three groups: female basketball $(\mathrm{r}=$ 0.772); male basketball ( $\mathrm{r}=-0.768)$; and male indoor soccer $(r=-0.712)$, for $\mathrm{p}<0.01$, and this good correlation could be considered to validate the test with distance adjustments between the plates. The intraclass correlation analyzed for the same population between the original test and the automated apparatus presented in Figure 5 shows satisfactory and significant results (0.901) for the validation of the AATT. In addition, the use of electronic equipment has been increasingly preferred with a view to avoid the interference that human evaluation may provoke (BalsalobreFrenadez et al., 2016; Garnacho-Castaño et al., 2015; González-Badillo \& Sánchez-Medina, 2010; Jidovtseff et al., 2011; Sanchez-Medina et al., 2014), being an important form of test control.

\section{CONCLUSION}

We can conclude from the present study that the automated apparatus for evaluation of upper limb velocity has been shown to be compatible 
with what it intends to evaluate, demonstrated that there is no significant difference between the results evaluated by the device and the original form in the same population. The use of the AATT presented advantages over the application of the original EUROFIT test applied manually, presenting a lower technical error of intra-rater measurement and it can be applied with only one evaluator, reducing the time required and the efficiency of the evaluations. Thus, it is an important and efficient piece of equipment that can be used by professionals for physical evaluations of children and adolescents in sports initiation.

\section{Acknowledgments:}

Nothing to declare.

\section{Conflict of interests:}

Nothing to declare.

\section{Funding:}

Nothing to declare.

\section{REFERENCES}

Alves, M., Ramos, D., Oliveira, G., Alves, H., Anderson, I., Magalhães, I. \& Ferreira, P.L. (2014). European Portuguese EARS test battery adaptation. Acta Medica Portuguesa, 27(1), 23-32.

Balsalobre-Fernández, C., Kuzdub, M., Poveda-Ortiz, P. \& Campo-Vecino, J. (2016). Validity and Reliability of the PUSH Wearable Device to Measure Movement Velocity During the Back Squat Exercise. Journal of Strength Conditioning Research, 30(7), 1968-74.

Brasile, F. (1990). Performance evaluation of wheelchair athletes: more than a disability classification level issue. Adapted Physical Activity Quarterly, 7, 289-97.

Brasile, F. (1986). Wheelchair basketball skills proficiencies versus NWBA classifications. Adapted Physical Activity Quarterly, 3, 6-13.

Cabral, B. G. A. T. (2013). Predictive equation of bone age in sports initiation through anthropometric variables. Brazilian Journal of Sport Medicine, 19(2), 99-103.

Dantas, M. P., Barbosa, P. R. S., Silva, L. M. \& Cabral, B. G. A. T. (2016) Relationship of bone and chronological age with physical abilities in sports initiation. Motricidade, 12(2), 28-34.

Eurofit Council of Europe. (1990). Commission for the Development of Sport, Eurofit: a test manual.
Falzon, C., Sabiston, C., Bergamaschi, A., Corrion, K., Chalabaev, A. \& D'Arripe-Longueville F. (2014) Development and validation of the Cancer Exercise Stereotypes Scale. Journal of Psychosocial Oncology, 32(6), 708-26.

Garnacho-Castaño, M. V., López-Lastra, S., Sanches, A. R. \& Mate-Muñoz, J. L. Reliability and validity assessment of a linear position transducer. Journal of Sports Science and Medicine, 14, 128-36.

González-Badillo, J. J. \& Sánchez-Medina, L. (2010). Movement velocity as a measure of loading intensity in resistance training. International Journal of Sports Medicine, 31, 347-352.

Jidovtseff, B., Harris, N. K., Crielaard, J. M. \& Cronin, J. B. (2011). Using the load-velocity relationship for 1RM prediction. Journal of Strength Conditioning Research, 25, 267-270.

Kilkens, O. J. E., Post, M. W. M., Dallmeijer, A. J., Seelem, H. A. M. \& Van Der Woulde L. H. V. (2003). Wheelchair Skills tests: a systematic review. Clinical Rehabilitation, 17, 418-30.

Krebs, R. J., Borges Junior, N. G. \& Santos, M. B. (2008). Implications of visual and proprioceptive perception in the performance of a continuous and closed motor task. Brazilian Journal of Motor Behavior, 1 (4), 35-9.

Krebs, R. J., Lucas, M. A. \& Saenz, G. C. Criteria for calculating the adjusted distances of the Eurofit battery. Cinergis, 1, 189-202.

Marques, E. A., Baptista, F., Santos, R., Vale, S., Santos, D. A. \& Silva, A. M. (2014). Normative functional fitness standards and trends of Portuguese older adults: cross-cultural comparisons. Journal of Aging Physical Activity, $22(1), 126-37$.

Neto Francisco, R., Xavier, R. F. C., Santos, A. P. M., \& Amaro, K. N., Florêncio, R., Poeta, L. S. (2013). Cross-laterality and reading and writing performance in schoolchildren. Cefac Journal, 15(4), 864-872.

Perini, T. A., Oliveira, G. L. , Ornellas, J. S. \& Oliveira, F. P. (2005). Calculation of the technical error of measurement in anthropometry. Brazilian Journal of Sports Medicine, 11(1), 81-85.

Rikli, R. E. \& Jones, C. J. (199). Development and validation of a functional fitness test for community residing older adults. Journal of Aging Physical Activity, 7(2), 129-61.

Sanchez-Medina, L., Gonzalez-Badillo, J. J., Perez, C. E. \& Pallares, J. G. (2014). Velocity- and power-load relationships of the bench pull vs. bench press exercises. International Journal of Sports Medicine, 35, 209-216.

Santos, M. B., Keulen, G. E., Braga, R. K., Dias, J. A., Afonso, C. A. \& Krebs RJ. (2007) System of evaluation of the speed of upper limbs. International Symposium on Sport Sciences; São Caetano Brazil.

Santos, M. B. (2009). Impact of a motor intervention program on the performance of schoolchildren in performing the upper limb agility test - Plate Strikes. Florianópolis: UDESC. 
Schmidt, R. \& Wrisberg, C. (2001) Learning and motor performance: a problem-based approach to learning. Porto Alegre: Artmed.

Wang, H. C., Chao, Y. F., Tsai, J. C., Yu, Y. H., Hang, L. W. \& Lee, S. D. (2014). Development and preliminar validation of a questionnaire on regular exercise beliefs among patients with chronic obstructive pulmonary disease. Journal of Clinical Nurse, 23(7-8), 1133-43.

Yilla, A. B., Sherril, C. (1998). Validating the Beck battery of quad rugby skills tests. Adapted Physical Activity Quarterly, 15(2), 155-67.

All content of Journal Motricidade is licensed under Creative Commons, except when otherwise specified and in content retrieved from other bibliographic sources. 